\title{
Cryostabilization of unwashed fish mince
}

\author{
CHONG M. LEE AND PEIZHI LIAN \\ Dept. of Nutrition and Food Sciences, College of the Environment and Life Sciences, \\ University of Rhode Island, Kingston, RI 02881, USA uricml@uriacc.uri.edu
}

SUMMARY: Mechanical refining and cryostabilization methods have been developed to increase the commercial use of fish mince from the underutilized fish species. Freeze-induced texture hardening and drip loss in the white, lean fish mince, and rancidity in the dark, oily fish mince can be controlled by the use of appropriate cryoprotectant mixtures. 2-4\% milk protein concentrate was effective in controlling rancidity and fishy odor developments in oily fish mince by binding low molecular weight reactive substances, while the combination of $2 \%$ soy protein concentrate and $0.4 \%$ alginate plus conventional cryoprotectants (4\% sorbitol, 0.3\% STPP) retarded fishy odor development and texture hardening in lean fish. Alginate helped maintain the dispersibility of fish mince by preventing muscle fiber interaction during frozen storage. Cryostabilization of fish mince enables the development of a variety of value-added mince-based seafood products formulated to provide desired flavor and texture.

\section{KEY WORDS: cryostabilization, fish mince, dispersibility, texture, proteins, alginate, mince- based seafood, mackerel, herring, red hake}

\section{INTRODUCTION}

Over the world, there are still many fish species, bycatches, and processing by-products which remain underutilized or unutilized. Our recent development of highly acceptable mince-based products (chedder cheese-flavored mackerel nugget and scampy sauceflavored herring nugget) led to an idea of separating refined meat and freeze stabilizing the mechanically recovered mince without washing for the production of a variety of value-added convenient seafood products, including products with a health concept such as a high omega-3 fatty acid product. The significance of this idea is to develop a new seafood base material and a manufacturing industry in a manner similar to the development of surimi and surimi industry. No previous attempts have been made to produce frozen stable fish mince blocks through refining without washing. The potential advantages of the proposed refining and cryostabilization process include no washing, hence no high BOD effluent, high yield, inventory control of frozen stable intermediate product to be used in formulated seafood products, and the process being inclusive of various fish species, by-catches, and processing by-products.

Problems and disadvantageous characteristics of frozen fish mince are associated with texture hardening or toughening, poor dispersibility (the condition in which the frozen-thawed mince is not easily dispersed when mixed with ingredients), poor functionality (e.g. gelling, formability, water holding ability), discoloration (darkening), lack of flaky texture, and lipid oxidation and fishy odor development.

A two-prong approach was made to cryostabilize oily pelagic and lean demersal fish using a combination of antioxidative proteins and cryoprotective polyols. The antioxidative role of proteins (skim milk, ${ }^{1)}$ wheat gliadin, ${ }^{2)}$ and soy protein isolate $^{3)}$ ) have been reported previously. The fishy odor development partly from rancidity is a primary concern in the pelagic fish mince, while the frozeninduced texture hardening is a primary problem with lean fish. For this reason, proteins were examined for pelagic fish mince, while cryoprotectants were evaluated for lean fish mince. In texture hardening of lean fish, calcium ion has been implicated since it may promote the formation of ionic cross-linkages between polypeptide chains ${ }^{4)}$ or catalyze an acyltransfer cross-linking reaction of myosin heavy chain by activating a $\mathrm{Ca}^{2+}$-dependent transglutaminase (TGase). ${ }^{5)}$ Thus, the removal of calcium ions by calcium chelating hydrocolloid such as alginate or carrageenan ${ }^{6)}$ may repress fish protein cross-linking formation. A combination of sorbitol, polyphosphates, and one of these biopolymers could control the development of texture hardening and poor dispersibility of frozen fish mince. However, the effects of protein additives and their combination with cryoprotectants on the physicochemical properties of minced fish muscle have not been previously studied. 


\section{MATERIALS AND METHODS}

Fresh Atlantic herring (Clupea harengus) and mackerel (Scomber scombrus) as representative pelagic fish species and red hake (Urophycis chuss) as demersal species were obtained from the local fish suppliers, Narragansett, RI. They were not more than two days old from the time of catch and kept in ice before processed within the same day. Herring and mackerel were manually filleted, while red hake were headed and gutted with thoracic bones removed before deboning.

\section{Preparation of refined mince}

The production of refined mince followed the general process illustrated below (Fig. 1).

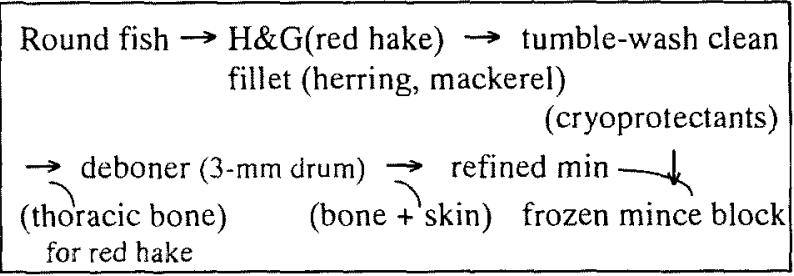

Fig. 1. Processing procedure for the preparation of refined mince.

The important points in designing the refining process are the process must be species specific; headed-and-gutted $(H \& G)$ with thoracic bone removed for the gadoid fish species; $H \& G$ for the mid-water species (e.g. bream); fillet for pelagic fish species; and the use of 3-mm drum. Thoracic bone is a thickest part of backbone in the cavity which releases blood and marrow upon mechanical compression during the deboning process. The inclusion of blood and marrow results in discoloration and quality deterioration due to lipid oxidation. Thoracic bones can be mechanically removed by a stamping-type cutter. A high-speed continuous filleting machine (Model 235) for herring and mackerel is available from Baader Machineries in Germany. A 3-mm drum is necessary to remove all scales and bone particles. A drum hole size smaller than $3 \mathrm{~mm}$ may destroy the integrity of muscle fiber resulting in the loss of fibrous texture. At the appropriate steps, the washing must be done adequately. The temperature of material must be kept at $10^{\circ} \mathrm{C}$ or below during the entire duration of refining to minimize deteriorative enzymatic as well as oxidative reactions.
Screening of cryoprotective additives for mackerel/herring and red hake mince

Mackerel and herring are oily pelagic fish and present a problem with lipid oxidation which leads to development of fishy odor and rancid taste. Unlike the lean gadoid fish, texture deterioration does not appear to be a problem in the pelagic fish. For this reason, antioxidative proteins, potato extract and cysteine were evaluated for their effectiveness in controlling rancidity and fishy odor development. Eleven (11) commercially available proteins, 1 potato extract (antioxidative), and 1 cysteine (free radical scavenging) were blended in mackerel mince at $2 \%$ level ( $0.2 \%$ for cysteine). The resulting mince were placed in the respective tin container $(60 \mathrm{~mm}$ dia $\times 18$ $\mathrm{mm}$ height; Elisco Inc., Baltimore, MD) covered with lids, packed in a nylon/polyethylene bag, and subjected to 4 freeze-thaw cycles. Each cycle consisted of freezing for 4 days at $-18^{\circ} \mathrm{C}$ and thawed at $4^{\circ} \mathrm{C}$ overnight. At the completion of 4 cycles, TBARS $^{\text {7) }}$ for the extent of lipid oxidation, and sensory and texture analyses were conducted. Texture and sensory analyses were done on cooked samples following the procedures described by Lian et al. ${ }^{8)}$ Upon screening with a 4 freeze-thaw cycle test, 6 proteins were selected out of 14 . They were soy protein isolate (ProFam 648, ADM, Decatur, IL), soy protein concentrate (SPC) (Arcon S, ADM), milk protein isolate (TMP-1350, New Zealand Milk Products, Santa Rosa, CA), milk protein concentrate (MPC) (Alapro 4560, NZMP), whey protein concentrate (Alacen 878, NZMP), and egg white (spary dried, Papettie Hygrade, Elizabeth, NJ). These six were incorporated at $4 \%$ into new batches of herring and mackerel mince and subjected to a 6 month frozen storage test at $-18 \mathrm{C}$ using the same evaluation parameters. Two most performance and cost effective proteins, namely, soy (Arcon S) and milk protein concentrates (Alapro), were selected and subjected to another round of 6-month frozen storage test using $0,2,4$, and $6 \%$ for determination of the respective optimum level in herring, mackerel and red hake mince.

Another screening was carried out for red hake mince to identify the best performing combination of cryoprotectants in addition to proteins. Because of its physicochemical susceptibility to freeze-induced textural changes, especially in the presence of calcium ions, $\mathrm{Ca}^{2+}$-chelating hydrocollolids, sorbitol and 
sodium tripolyphosphate (STPP) were included in addition to protein. The cryoprotectant combinations studied were $2 \%$ SPC-4\% sorbitol- $0.3 \%$ STPP, $0.4 \%$ alginate- $4 \%$ sorbitol- $0.3 \%$ STPP, iota-carrageenan$4 \%$ sorbitol- $0.3 \%$ STPP, and $0.2 \% \quad$ SPC- $0.4 \%$ alginate-4\% sorbitol-0.3\% STPP, where alginate (Keltone) and iota-carrageenan (Gelcarin, GP 359) were supplied by Kelco (San Diego, CA) and FMC (Philadelphia, PA), respectively. Each mince with respective cryoprotectant mixture received the same preparation procedure for screening of proteins as described previously and stored frozen at $-18^{\circ} \mathrm{C}$ for 0 , 4,8 and 17 weeks. One sample from each treatment was kept at $-84^{\circ} \mathrm{C}$ as a reference. An approximately $300 \mathrm{~g}$ mince block $(15 \times 13 \times 1.3 \mathrm{~cm})$ from each treatment was also stored frozen for 17 weeks for the dispersibility test.

\section{Measurements of texture changes}

For uncooked samples, frozen fish mince was thawed at $4^{\circ} \mathrm{C}$ for $12 \mathrm{~h}$, while cooked samples were prepared by steaming thawed samples in a $90^{\circ} \mathrm{C}$ steam-cooker for $10 \mathrm{~min}$ and holding overnight in a $4^{\circ} \mathrm{C}$ walk-in refrigerator. Both were held at approximately $22^{\circ} \mathrm{C}$ for $1 \mathrm{~h}$ before texture analysis. Texture evaluation of cooked or uncooked frozen fish mince was performed on the Instron Universal Testing Machine (Model 1122; Instron Engineering Corp., Canton, MA) while the sample was in the metal container. Penetration force at $30 \%$ deformation at $50 \mathrm{~mm} / \mathrm{min}$ was used to measure the resistance to penetration using an $8-\mathrm{mm}$ diameter stainless steel plunger with a flat end. Shear force was measured by shearing sample perpendicularly with a flat rectangular blade ( $1 \mathrm{~mm}$ thickness $\times 26 \mathrm{~mm}$ width $\times 40 \mathrm{~mm}$ height) to $70 \%$ deformation at $100 \mathrm{~mm} / \mathrm{min}$. The first peak was used to measure the hardness that resulted from freezeinduced muscle fiber association.

\section{Determination of dispersibility}

The dispersibility of frozen-thawed red hake mince samples of $4^{\circ} \mathrm{C}$ was evaluated as the ease of dispersing the thawed mince $(300 \mathrm{~g})$ during mixing for $1 \mathrm{~min}$ in a Kitchen-Aid bowl mixer at a setting of "5" ( 1 very difficult -9 very easy to mix, mashed potato used for 9).

\section{Statistical analysis}

The experiment was designed to compare additives for their effects on physicochemical properties of fish mince during frozen storage. The Tukey's test was performed to identify significant differences $(\mathrm{P}<0.05)$ between means corresponding to different treatments using the Systat version 5.2 (Systat Inc., Evanston, IL).

\section{RESULTS AND DSICUSSION}

Results of a 6-month frozen storage test of two most performance and cost effective proteins, SPC and MPC, are given in Table 1. The most effective level was $4 \%$ with the minimum level at $2 \%$. An interesting finding was that MPC (lower TBARS, fishy odor and penetration force) was suitable for pelagic fish mince while SPC better suited to red hake mince. After a 6month frozen storage of red hake mince, there was no difference in fishy odor score between MPC (score 1) and SPC (score 1). However, the differences were observed in hardness (SPC:6.5; MPC:8.5 1 soft -9 hard) and texture desirability (SPC:5; MPC:2 1 undesirable - 9 very desirable). The different responses (TBARS and fishy odor) of pelagic fish muscle to MPC and SPC could be explained by the characteristic binding between protein and low molecular weight reactive substances in the muscle. This postulation is supported by a review article on interaction of flavor with proteins. ") The texture moderating effect of soy protein was due to its

Table 1. Changes in TBARS, fishy odor and penetration force of uncooked mackerel mince stored frozen for 6 months at varying levels of added proteins

\begin{tabular}{|c|c|c|c|c|c|c|c|c|c|c|c|c|}
\hline & \multicolumn{4}{|c|}{ TBARS $($ umole/100g) } & \multicolumn{4}{|c|}{ FISHY ODOR SCORE* } & \multicolumn{4}{|c|}{ PENETRATION FORCE (g) } \\
\hline & 0 & 2 & 4 & $6 \mathrm{mo}$ & 0 & 2 & 4 & $6 \mathrm{mo}$ & 0 & 2 & 4 & $6 \mathrm{mo}$ \\
\hline Control & 3.2 & 1.89 & 2.73 & 4.15 & 4 & 4 & 5 & 5 & 785 & 835 & 918 & 1069 \\
\hline MPC- $2 \%$ & 2.06 & 1.25 & 2.41 & 4.24 & 2 & 3 & 2 & 2 & 903 & 985 & 1169 & 1013 \\
\hline $\mathrm{MPC}-4 \%$ & 1.88 & 1 & 2.16 & 3.35 & 2 & 2 & 1 & 1 & 838 & 963 & 1123 & 894 \\
\hline MPC. $6 \%$ & 2.2 & 1.23 & 1.98 & 2.89 & 2 & 2 & 1 & 1 & 898 & 980 & 1125 & 813 \\
\hline SPC-2\% & 3.19 & 1.49 & 2.76 & 4 & 2 & 3 & 2 & 2 & 1125 & 1138 & 1285 & 1131 \\
\hline SPC- $4 \%$ & 3.13 & 1.46 & 3.01 & 5.2 & 2 & 3 & 2 & 2 & 1161 & 1305 & 1303 & 1388 \\
\hline SPC- $6 \%$ & 3.17 & 1.53 & 3.7 & 5.51 & 2 & 3 & 2 & 2 & 1305 & 1500 & 1510 & 1438 \\
\hline
\end{tabular}

* I none -9 very strong; MPC: milk protein concentrate; SPC: soy protein concentrate 
physical interference with muscle fiber interaction such as crosslinking between protein molecules. ${ }^{1(1)}$ The addition of proteins with a minimum of $2 \%$ controlled the development of fishy odor, a sign of oxidation of low molecular reactive compounds including fatty acids in both red hake and pelagic fish. This is believed to be a result of protein binding with such reactive compounds making them less prone to oxidative reactions responsible for the development of fishy odor.

The dispersibility of fish mince is an important quality requirement for the ease of mixing with other ingredients during formulation. The high dispersibility value ( 7.3 vs 1.8 for control) indicates that $0.4 \%$ alginate $-4 \%$ sorbitol $-0.3 \%$ STPP mixture effectively controlled the freeze-induced muscle compaction in red hake mince during the 16-week frozen storage. An improvement in dispersibility was accompanied by concurrent reductions in penetration $(400 \mathrm{~g}$ to $100 \mathrm{~g})$ and shear forces $(1125$ $\mathrm{g}$ to $150 \mathrm{~g}$ ) of uncooked mince. Sorbitol and STPP with or without SPC and carrageenan was not as effective as alginate mixture indicating that it did not chelate calcium ions nor sufficiently inhibited the interaction of muscle fibers. The preventative role of alginate in texture hardening is believed to be related to its ability to chelate calcium ions responsible for crosslinking and interfere with muscle fiber interaction. ${ }^{8)}$

The calcium content (measured by AA spectrophotometry) ${ }^{11)}$ in red hake $(29.4 \mathrm{mg} / 100 \mathrm{~g})$ was found to be significantly greater than Alaska pollock (16.5) and Pacific whiting (8.7), ${ }^{12)}$ suggesting that it plays a key role in texture hardening of fish mince since uncooked red hake mince ( 400 g penetration force) became significantly harder than Alaska pollock mince $(150 \mathrm{~g})$.

The total free - SH content in the control red hake mince significantly decreased over the 17-wk frozen period. ${ }^{8)}$ This was consistent with the reduction of SDS-soluble protein, implying the involvement of the formation of disulfide bond in protein insolubilization of frozen mince. These findings were further supported by the result of SDS-PAGE ${ }^{\text {() }}$ After $17 \mathrm{wk}$ of frozen storage, no change in myosin heavy chain (MHC) band of $0.4 \%$ alginate- $4 \%$ sorbitol- $0.3 \%$ STPP was observed while a noticeable reduction in $\mathrm{MHC}$ of control indicating that MHC in the control underwent polymerization through S-S crosslinking.

\section{CONCLUSION}

The development of fishy odor and rancidity in oily pelagic fish mince was effectively controlled by addition of milk protein concentrate at $2-4 \%$, while freeze-induced textural hardening and poor dispersibility in red hake mince were effectively retarded by addition of $2 \%$ SPC- $0.4 \%$ alginate- $4 \%$ sorbitol-0.3\% STPP. Cryostabilization of fish mince will enable the development of a variety of valueadded mince-based seafood products formulated to provide desired flavor and texture.

\section{ACKNOWLEDGEMENTS}

Contribution No. 3909 of the College of the Environment and Life Sciences, University of Rhode Island, with the financial support from the Rhode Island Agricultural Experiment Station and the National Marine Fisheries Service, NOAA.

\section{REFERENCES}

1. Taylor MJ, Richardson T. Antioxidant activity of skim milk: effect of heat and resultant sulfhydryl groups. $J$. Dairy Sci. 1980; 63: 1783-1795.

2. Taguchi $K$, Iwami $K$, Kawabata $M$, lbuki $F$. Antioxidant effects of wheat gliadin and hen's egg white in powder model systems: protection against oxidative deterioration of safflower oil and sardine oil. Agric. Biol. Chem. 1988; 52: 539-545.

3. Wu SY, Brewer MS. Soy protein isolate antioxidant effect on lipid peroxidation of ground beef and microsomal lipids. J. Food Sci. 1994; 59: 702-706, 738.

4. Sikorski ZE, Olley J, Kostuch S. Protein changes in frozen fish. CRC Cric. Rev. Food Sci. Nutr. 1976; 8(1): 97-129.

5. Lee N, Park JW. Calcium compounds to improve gel functionality of Pacific whiting and Alaska pollock surimi. J. Food Sci. 1998; $63: 969-974$.

6. Glicksman $M$, King AH. Seaweed extracts. In Glicksman M (ed) Food Hydrocolloids, vol. IIl CRC Press, Boca Raton, FL, 1983, pp.115-188.

7. Lemon DW. An improved TBA test for rancidity 1975; Halifax Lab. New Ser. Circular No. 51.

8. Lian PZ, Lee CM, Hufnagel L. Physicochemical properties of frozen red hake (Urophycis chuss) mince as affected by cryoprotective ingredients. J. Food Sci. 2000; 65: 1117-1123.

9. Fisher $\mathrm{N}$, Widder $\mathrm{S}$. How proteins influence food flavor. Food Technol. 1997; 51(1): 68-70.

10. Yoon KS, Lee CM, Hufnagel LA. Textural and microstructural properties of frozen fish mince as affected by the addition of nonfish proteins and sorbitol Food Structure 1991; 10: 255-265.

11. Osborne DR, Voogt P. The analysis of nutrients in foods. 1978, Academic Press, London.

12. Park JW. New developments in surimi and surimi seafood. 11th World Congr. Food Sci. Technol. Seoul Korea, April 22-27. 